\title{
Time since rituximab treatment is essential for developing a humoral response to COVID-19 mRNA vaccines in patients with rheumatic diseases.
}

Running title: Rituximab and COVID-19 vaccine

Anne Troldborg, MD, $\mathrm{PhD}^{1,2,3}$ (https://orcid.org/0000-0002-9501-1268)

Marianne Kragh Thomsen, MD 3,5 (https://orcid.org/0000-0002-3645-6689)

Lars Erik Bartels, MD, PhD ${ }^{1}$ (https://orcid.org/0000-0002-9101-1076)

Jakob Bøgh Andersen, MD ${ }^{1}$ (https://orcid.org/0000-0003-0189-4096)

Signe Risbøl Vils MS ${ }^{1,2}$ (https://orcid.org/0000-0002-9212-921X)

Clara Elbæk Mistegaard, MD ${ }^{1,2,5}$

Anders Dahl Johannsen, MD $^{1}$ (https://orcid.org/0000-0001-5975-9394)

Marie-Louise From Hermansen, MD, $\mathrm{PhD}^{1}$ (https://orcid.org/0000-0002-9108-8604)

Susan Mikkelsen, $\mathrm{MD}^{4}$ (https://orcid.org/0000-0001-7806-1143)

Christian Erikstrup, MD, $\mathrm{PhD}^{3,4}$ (https://orcid.org/0000-0001-6551-6647)

Ellen-Margrethe Hauge, MD, PhD ${ }^{1,3}$ (https://orcid.org/0000-0003-2562-9174)

Christian Ammitzbøll, MD, $\mathrm{PhD}^{1,3}$ (https://orcid.org/0000-0002-8535-1613).

1: Department of Rheumatology, Aarhus University Hospital, Aarhus, Denmark

2: Department of Biomedicine, Aarhus University, Aarhus, Denmark

3: Department of Clinical Medicine, Aarhus University, Aarhus, Denmark

4: Department of Clinical Immunology, Aarhus University Hospital, Aarhus, Denmark

5: Department of Clinical Microbiology, Aarhus University Hospital, Aarhus, Denmark

Conflicts of interest: none.

Financial support: The Danish Rheumatism Association

Corresponding author:

Anne Troldborg

Department of Rheumatology

Palle Juul-Jensens Boulevard 99

8200 Aarhus N

Denmark

annetrol@rm.dk 


\begin{abstract}
Objective

We aimed to investigate if patients with rheumatic diseases treated with rituximab raise a serological response towards the COVID-19 mRNA vaccines and to elucidate the influence of time since the last dose RTX before vaccination on this response.
\end{abstract}

Methods

We identified and included 201 patients with rheumatic diseases followed at the out-patient clinic at the Department of Rheumatology, Aarhus University Hospital, who had been treated with RTX in the period 2017-2021 and who had finished their two-dose vaccination with a COVID-19 mRNA vaccine. Total antibodies against SARS-CoV2 spike protein were measured on all patients and 44 blood donors as a reference.

\title{
Results
}

We observed a time-dependent increase in antibody response as the interval from the last RTX treatment to vaccination increased. Only $17.3 \%$ of patients developed a detectable antibody response after receiving their vaccination 6 months or less after their previous RTX treatment. Positive antibody response increased to $66.7 \%$ in patients who had RTX 9-12 months before vaccination. All blood donors $(100 \%)$ had detectable antibodies after vaccination.

\section{Conclusion.}

Patients with rheumatic diseases treated with rituximab have a severely impaired serological response towards the COVID-19 mRNA vaccine. Our data suggest that the current recommendations of a 6 months interval between rituximab treatment and vaccination should be revised. 


\section{Introduction}

Reports of increased risk of a severe outcome and death of COVID-19 in patients treated with Bcell depleting therapy, like rituximab (RTX) (1-3), have raised particular concern for patients with rheumatic diseases (RD) receiving this treatment. These data favor prioritizing vaccination of patients treated with RTX.

B-cell depletion is an established option in a wide range of rheumatic diseases, such as rheumatoid arthritis, myositis, and vasculitis. However, B-cells are crucial for the induction of protective immunity after vaccination and infection.

Since patients receiving immune inhibiting medication were largely excluded from COVID-19 phase 3 vaccine trials, there is limited data on the effectiveness of vaccination in patients treated with RTX. The primary concern about RTX treatment is the risk of reduced immunogenicity to COVID-19 vaccination analogous to what has been observed with, i.e., the influenza vaccination (4). However, existing knowledge extrapolated from experiences with other vaccines may not translate to the novel mRNA vaccines available for COVID-19.

In our preliminary data of patients with RD receiving the Pfizer/BioNTech mRNA vaccine, we observed that only four out of 17 (23.5\%) receiving RTX had measurable antibodies against SARS-CoV-2 after vaccination (5). These findings have lately been confirmed by several other research groups (6-8)

Although the half-life of rituximab is approximately 20 days, the recovery of the B cell count typically starts at 6 to 9 months after the last treatment, and normal levels are obtained after 9-12 months (9). Thus, RTX treatment may markedly and enduringly debilitate the humoral response to vaccination, and therefore most rheumatology guidelines recommend waiting for 6 months after rituximab infusion before vaccinating (10).

Concerns about RTX treatment and potentially inadequate vaccine response were aired early in the pandemic (11), and initial data seem to support this concern $(5,12-14)$. So far, most studies 
regarding patients with RDs and the COVID-19 vaccines have focused on various treatments and included a relatively small number of patients receiving rituximab.

The objective of this study was to investigate if patients with rheumatic diseases treated with rituximab raise a serological response towards the COVID-19 mRNA vaccines and to elucidate the influence of time since the last dose RTX before vaccination on this response.

\section{Materials and methods}

\section{Patients}

We identified all patients currently followed at the out-patient clinic at the Department of Rheumatology, Aarhus University Hospital (AUH), who had received RTX treatment since 2017. All patients with a systemic autoimmune rheumatic disease, who had received a two-dose mRNA vaccination, were eligible for inclusion had they received RTX in the specified period. We identified 308 individuals, of whom 257 had finished vaccination with two doses of an mRNA vaccine with three weeks between the first and the second dose (suppl. fig. 1). Out of the 257 vaccinated individuals, 201 gave their informed consent to participate in the study and had antibodies measured.

Patients received their vaccines between January and June of 2021. All patients followed the same vaccination schedule managed by the Danish National Health Authorities, and received two doses of an mRNA vaccine with three weeks between the first and the second dose.

As a reference for a normal serological response, we included a randomly selected group of blood donors (BD) $(n=44)$ from the Blood Bank, AUH, who had received two doses of a COVID-19 mRNA vaccine.

All included patients were asked at inclusion whether they had tested positive for SARS-CoV-2 prior to vaccination (PCR test or nasal swap quick test).

\section{SARS-CoV-2 antibody testing}

We aimed at measuring antibodies 4-6 weeks after the second vaccination at the expected time for highest antibody levels. 
Serum total antibodies against recombinant SARS-CoV-2 spike S1 protein were measured in a commercially available assay (VITROS Immunodiagnostic Products Anti-SARS-CoV-2 Total test, Ortho Clinical Diagnostics, Rochester, NY, USA). According to the manufacturer's instructions, the analyzes were performed at the Department of Clinical Microbiology, AUH, by experienced staff.

The assay is a semi-quantitative SARS-CoV-2 double antigen sandwich chemiluminescent immunoassay. It detects total antibodies captured by recombinant SARS-CoV-2 spike S1 protein coated in a microtiter well. A one-level calibration is lot-specific and links the sample signal (S) to a cut-off value $(\mathrm{CO})$. Signal/cut-off $(\mathrm{S} / \mathrm{CO}) \geq 1$ was considered positive, and $\mathrm{S} / \mathrm{CO}<1$ was considered negative. Results were based on a single test result.

Performance characteristics of the assay have been determined in a Danish validation study (15). The assay has a sensitivity of $95.3 \%$ (95\% confidence interval (CI): 90.7 - 97.7) and a specificity of $100 \%$ (95\% CI: 99.4 - 100). No cross-reactivity was observed.

\section{Statistics}

Unless otherwise stated, all values reported are medians with interquartile range (IQR). Univariate and multivariate logistic regression analyses were performed with presence of SARSCoV-2 antibodies after vaccination as the dependent variable. We included explanatory variables in the univariate model based on previous knowledge of association to vaccination response (age, gender, diagnosis and treatment) and to evaluate the effect of RTX treatment (time from last RTX to vaccination, total dose of RTX, total number of RTX infusions, total treatment time of RTX). Finally, time from vaccination to blood sample was included, as antibody levels wary over time. We did not correct for multiple hypothesis testing, as this was an exploratory design. We only included explanatory variables in the multivariate model that were significant in the univariate models.

Differences in antibody concentration were tested with Mann-Whitney U test.

\section{Ethics}


The study was approved by the Central Denmark Region Committee on Health Research Ethics (1-10-72-238-21) and the regional Danish Data Protection Agency (1-16-02-254-21). All patients and controls were offered participation in the study after informed consent. The study was conducted according to the Helsinki declaration.

\section{Results}

Two-hundred-and-one patients (78\% of eligible patients) were included in the study. Patients were predominantly female $(\mathrm{F} / \mathrm{M}=135 / 66)$ with a mean age of 58.4 years (SD 14.2). The most frequent diagnosis was ANCA-associated vasculitis $(n=65,32 \%)$, rheumatoid arthritis $(n=63$, $31 \%)$, and myositis $(n=28,14 \%)$. Seven patients had tested positive for SARS-CoV-2 prior to vaccination. Patient characteristics can be found in table 1 and in supplementary table 2 . The mean age of $\mathrm{BD}$ was $42(\mathrm{SD}=12.6)$, and they were predominantly female $(\mathrm{F} / \mathrm{M}=36 / 8)$.

We observed a time-dependent increase in antibody response as the interval from the last RTX treatment to vaccination increased (Figure 1A). Eighty-one patients had received RTX within 6 months prior to vaccination, and only $17.3 \%$ of these patients $(14 / 81)$ had a detectable antibody response. Positive antibody response increased to $66.7 \%$ in patients who had RTX 9-12 months before vaccination. This was comparable to patients who had received RTX 18-60 months before vaccination $(65.9 \%, 29 / 44)$ (Figure 1A). These results were independent of age, gender, diagnosis, cumulative RTX dose, and cumulative RTX treatment time but were influenced by prednisone and azathioprine treatment in a multivariable logistic regression analysis (Table 2). All BD (100\%) had a detectable antibody response after vaccination.

Patient total antibody concentration (median $111 \mathrm{AU} / \mathrm{ml}$ ) was significantly lower than the BD concentration (median $916 \mathrm{AU} / \mathrm{ml})(\mathrm{p}<0 \cdot 001$, figure 1B and suppl. table 1). This difference was significant even when the last RTX was given 18-60 months prior to vaccination (123 AU/ml) $(p<0 \cdot 001)$. In addition, the antibody response was lower in all rheumatic diagnoses compared to BD (suppl. fig. 2). No association between age and serological response was observed in our cohort (data not shown). 
The median time between the second vaccination and antibody measurement was 42 (IQR 1264) days for the patients and 33 (IQR 13-52) days for the BD.

\section{Discussion}

We report the results of the most extensive single-center study on patients with rheumatic diseases treated with RTX during the COVID pandemic receiving a two-dose vaccination with an mRNA COVID-19 vaccine. The study confirms a significantly impaired humoral response to mRNA vaccines in patients receiving RTX. Further, that ample time from last rituximab infusion to time of vaccination is pivotal in developing a humoral response after vaccination.

Data from other studies on patients with RDs have concordantly observed lower serological response in patients treated with RTX $(6-8,13,14)$, and similar data has been reported in other diseases where B-cell-depleting therapy is used $(16,17)$. When compared to BD the level of antibodies is generally lower in the patients. This is likely due to the immunosuppressive treatment (18); however, it could also be due to the autoimmune disease itself. In our univariate and multivariate model both prednisolone and azathioprine seemed to have an impact on the vaccine response.

The specific consequences of reduced antibodies are unknown, as we still lack knowledge of how to accurately measure immunity to SARS-CoV-2 infection, which may include, i.e., antibody level, antibody type, and T cell memory. In a recent study, Prendecki et al. demonstrate that B-cell depletion following RTX impairs serological responses, but T-cell responses are preserved. Thus, the absence of a serological response does not preclude T-cell mediated immunity. Whether a secluded T-cell response is sufficient to protect patients without a serological response remains to be investigated. However, even though a positive antibody response does not necessarily mean protection against COVID-19 disease, undetectable antibodies after vaccination pose an immediate concern since a serological response against SARS-CoV-2 is essential in the early phases of the infection (19). 
Our findings of an increased serological response 9-12 months after RTX treatment are in line with data showing the recovery of the B-cell-count in most cases starts at 6 to 9 months after the last treatment, and normal levels are obtained after 9-12 months (9).

Bonelli et al. observed a humoral immune response in RD patients with measurable peripheral B cells following RTX treatment (13). This could pose a method of identifying the patients likely to respond to vaccination, although more extensive studies are needed to confirm these findings.

An important finding in this study is that neither cumulative treatment time nor cumulative RTX dose seemed to influence the serological response to the vaccine. Thus, even in patients who have received RTX for a substantial time, expanding time-since-last-RTX treatment could prove beneficial for increasing the chance of a serological response.

The limitations of this study include lack of a quantitative assay to measure antibody serum concentrations. In addition, characterization of memory B- and T-cells were not available, and neither was data on repopulation of B-cells at the time of vaccination. Further, there was a lack of serial measurements of antibodies and knowledge of disease activity in patients at the time of vaccination. Lastly, matching between patients and controls by age was not possible.

However, this is the first study to examine a large cohort of RD patients treated with RTX, and our results indicate that time since last RTX treatment is a crucial element in mounting a serological response. Our data warrant development of strategies to increase vaccine-induced immunogenicity in patients treated with RTX. Measuring peripheral B-cells at the time of vaccination or identifying patients where postponing RTX or changing immunomodulatory therapy is clinically feasible could be strategies to increase immunogenicity.

In conclusion, patients with rheumatic diseases treated with RTX have a severely impaired serological response towards the COVID-19 mRNA vaccine. This is especially true if the interval between RTX treatment and vaccination is less than 9 months.

For the majority of RTX treated patients, the recommended six months since last RTX is insufficient to develop a humoral response to COVID-19 mRNA vaccines, and our data suggest that the current recommendations of a 6 months interval should be re-evaluated. 


\section{References}

1. Avouac J, Drumez E, Hachulla E, et al. COVID-19 outcomes in patients with inflammatory rheumatic and musculoskeletal diseases treated with rituximab: a cohort study. Lancet Rheumatol 2021;3:e419-e426

2. Strangfeld A, Schäfer M, Gianfrancesco MA, et al. Factors associated with COVID-19related death in people with rheumatic diseases: Results from the COVID-19 Global Rheumatology Alliance physician-reported registry. Ann Rheum Dis 2021;80:930-42.

3. Bachiller-Corral J, Boteanu A, Garcia-Villanueva MJ, et al. Risk of severe coronavirus infection (COVID-19) in patients with inflammatory rheumatic diseases. J Rheumatol 2021;48:1098-1102.

4. Westra J, van Assen S, Wilting KR, et al. Rituximab impairs immunoglobulin (Ig)M and IgG (subclass) responses after influenza vaccination in rheumatoid arthritis patients. Clin Exp Immunol 2014;178:40-7.

5. Ammitzbøll C, Bartels LE, Andersen JB, et al. Impaired antibody response to the BNT162b2 mRNA COVID-19 vaccine in patients with Systemic Lupus Erythematosus and Rheumatoid Arthritis. ACR Open Rheumatol 2021;3:622-628.

6. Magliulo D, Wade SD, Kyttaris VC. Immunogenicity of SARS-CoV2 vaccination in rituximab-treated patients: Effect of timing and immunologic parameters. Clin Immunol. $2021 ; 234: 108897$.

7. Ferri C, Ursini F, Gragnani L, et al. Impaired immunogenicity to COVID-19 vaccines in autoimmune systemic diseases. High prevalence of non-response in different patients' subgroups. J Autoimmun. 2021;125:102744.

8. Avouac J, Miceli-Richard C, Combier A, et al. Risk factors of impaired humoral response to COVID-19 vaccination in rituximab treated patients. Rheumatology 2021; Online ahead of print.

9. Thiel J, Rizzi M, Engesser M, et al. B cell repopulation kinetics after rituximab treatment in ANCA-associated vasculitides compared to rheumatoid arthritis, and connective tissue diseases: a longitudinal observational study on 120 patients. Arthritis Res Ther 2017;19:101. 
10. Furer V, Rondaan C, Heijstek MW, et al. 2019 update of EULAR recommendations for vaccination in adult patients with autoimmune inflammatory rheumatic diseases. Ann Rheum Dis 2020;79:39-52.

11. Houot R, Levy R, Cartron G, Armand P. Could anti-CD20 therapy jeopardise the efficacy of a SARS-CoV-2 vaccine? Eur J Cancer 2020;136:4-6.

12. Prendecki M, Clarke C, Edwards H, et al. Humoral and T-cell responses to SARS-CoV-2 vaccination in patients receiving immunosuppression. Ann Rheum Dis 2021;80:13221329.

13. Bonelli MM, Mrak D, Perkmann T, Haslacher H, Aletaha D. SARS-CoV-2 vaccination in rituximab-treated patients: Evidence for impaired humoral but inducible cellular immune response. Ann Rheum Dis 2021:80:1355-1356.

14. Furer V, Eviatar T, Zisman D, et al. Immunogenicity and safety of the BNT162b2 mRNA COVID-19 vaccine in adult patients with autoimmune inflammatory rheumatic diseases and in the general population: a multicentre study. Ann Rheum Dis 2021;80:1330-1338.

15. Harritshøj LH, Gybel-Brask M, Afzal S, et al. Comparison of sixteen serological SARSCoV-2 immunoassays in sixteen clinical laboratories. J Clin Microbiol 2021;59:11.

16. Herishanu Y, Avivi I, Aharon A, et al. Efficacy of the BNT162b2 mRNA COVID-19 vaccine in patients with chronic lymphocytic leukemia. Blood 2021;137:3165-73.

17. Gallo A, Capuano R, Donnarumma G, et al. Preliminary evidence of blunted humoral response to SARS-CoV-2 mRNA vaccine in multiple sclerosis patients treated with ocrelizumab. Neurol Sci. 2021;42:3523-3526.

18. Park JK, Lee YJ, Shin K, et al. Impact of temporary methotrexate discontinuation for 2 weeks on immunogenicity of seasonal influenza vaccination in patients with rheumatoid arthritis: a randomised clinical trial. Ann Rheum Dis 2018;77:898-904.

19. McMahan K, Yu J, Mercado NB, et al. Correlates of protection against SARS-CoV-2 in rhesus macaques. Nature 2021;590:630-4. 


\section{Figure and table legends}

Figure 1. Total SARS-CoV-2 antibody response after receiving two vaccinations with COVID19 mRNA vaccine in patients with rheumatic diseases treated with rituximab (blue circles) and controls (red crosses) . A) Percent of patients and controls with a measurable antibody response post-vaccination. B) Levels of measured total anti-SARS-CoV-2 antibodies in patients and controls. The $\mathrm{X}$-axis shows time since the patients' last treatment with rituximab prior to vaccination.

$\mathrm{n}=$ number of individuals with SARS-CoV-2 antibodies, $\mathrm{N}=$ total number of individuals. Blood donors (BD)

Table 1. Patient demographics. Interquartile range (IQR), Granulomatosis with polyangiitis (GPA), Eosinophilic Granulomatosis with polyangiitis (EGPA), \#Time between the first and last Rituximab treatment before vaccination.

Table 2. Univariate logistic regression analysis of antibody response after COVID-19 mRNA vaccination in patients.

Explanatory variables in the univariate model are based on previous knowledge of association to vaccination response and evaluation of RTX exposure. Specific DMARD treatments were included if $\geq 10 \%$ of the patients received it. The multivariate model includes all explanatory variables with statistically significant effects in the univariate analysis. "Time from last RTX treatment to vaccination" and "Prednisone treatment" were included as a continuous variables. Confidence interval (CI), odds ratio (OR), Granulomatosis with polyangiitis (GPA), eosinophilic Granulomatosis with polyangiitis (EGPA), disease-modifying anti-rheumatic drug (DMARD). 

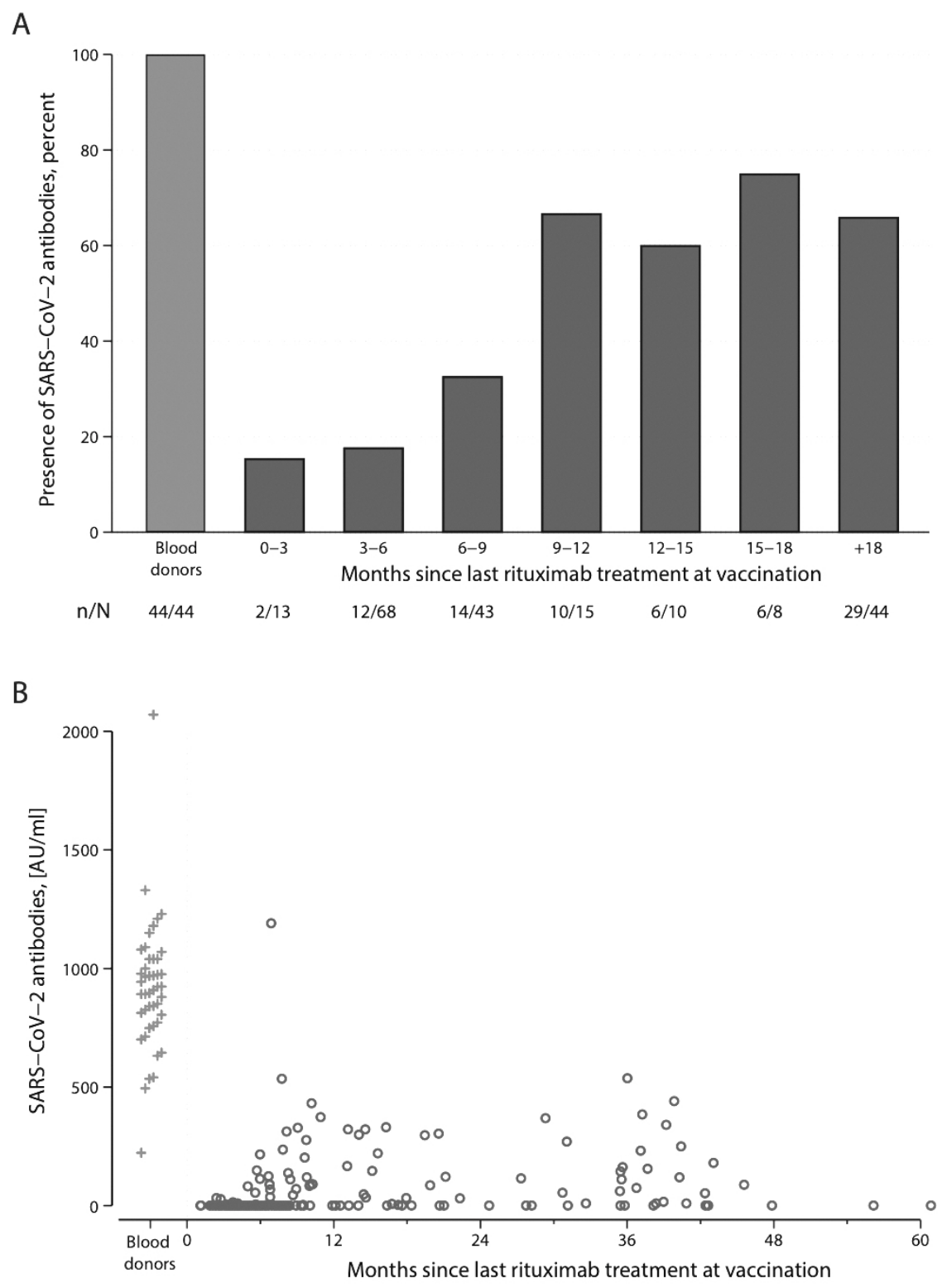

Figure 1

$191 \times 241 \mathrm{~mm}(144 \times 144$ DPI $)$ 
Female sex, no(\%)

Age, mean (SD)

(

Disease duration, years

Diagnosis

Rheumatoid Arthritis

63

Systemic Lupus Erythematosus

20

ANCA-vasculitis, GPA/EGPA

$61 / 4$

Poly- Dermatomyositis

28

Scleroderma

10

Other diagnosis

14

$(3.0-8.0)$

mRNA vaccine used, no (\%)

Pfizer/Biontec

195

Moderna

6

42

$31 \%$

$10 \%$

$32 \%$

$14 \%$

$5 \%$

$7 \%$

Time from vaccination to blood sample, days

$97 \%$

$3 \%$

DMARD treatment

None

52

$(12-64)$

Prednisone

Median prednisone dose, mg (IQR)

84

$25 \%$

5

$42 \%$

Methotrexate

51

Hydroxychloroquine

22

Azathioprine

21

Leflunomide

11

Mycophenolate

Immunoglobuline

Cyclophosphamide

Salazopurine

Biologic treatment

None

Rituximab - within last 15 months

TNF-inhibitors

JAK-inhibitors

IL-6-inhibitors

Abatacept

Previous rituximab treatment

Number of infusions

Cummulativ total dose, $\mathrm{mg}$

Total treatment time\#, days

9

6

2

1

(5-10)

$25 \%$

$11 \%$

$10 \%$

$5 \%$

$4 \%$

$3 \%$

$1 \%$

$0.5 \%$

35

$17 \%$

145

$72 \%$

6

$3 \%$

$3 \%$

$2 \%$

5

$2 \%$

5

$(2-8)$

4000

$(2000-8000)$

505

(30 - 1532)

Table 1. 
Univariate logistic regression analysis

Sex, female $=$ ref.

Age, years

Time from vaccination to blood sample

Diagnosis

Rheumatoid Arthritis

Systemic Lupus Erythematosus

ANCA-vasculitis (GPA/EGPA)

Poly- Dermatomyositis

Scleroderma

Other diagnosis

Rituximab exposure

Rituximab treatment within last 15 months

Months between last Rtx and vaccination

Total number of Rituximab infusions

Total dosis of Rituximab, mg

Time from first to last Rituximab treatment

DMARD treatment

No treatment

Prednisone treatment, (yes/no) no=ref

Prednisone treatment, dose in $\mathrm{mg}$

Methotrexate treatment

Hydroxychloroquine treatment

Azathioprine

Multiple logistic regression analysis

Months between last Rtx and vaccination

Prednisone treatment, $\mathrm{mg}$

Azathioprine treatment
OR

0.91

\section{$95 \% \mathrm{CI}$}

$0.50-1.67$

$0.96-1.00$

$0.99-1.01$

1.00

- ref-

0.57

0.83

0.36

1.33

2.67

0.21

1.06

0.96

0.97

1.00

0.94

0.49

0.91

1.71

0.87

0.23

1.08

0.91

0.10
- ref-

$$
0.19-1.69
$$

$0.41-1.69$

$0.13-1.02$

$0.35-5.07$

$0.82-8.71$

$0.10-0.40$

$1.03-1.09$

$0.92-1.01$

$0.92-1.02$

$1.00-1.00$

$0.50-1.78$

$0.27-0.89$

$0.85-0.99$

$0.90-3.25$

$0.35-2.19$

$0.06-0.80$

$1.04-1.11$

$0.84-0.99$

$0.02-0.44$
P-value

0.77

0.09

0.61

- ref-

0.31

0.61

0.06

0.67

0.10

$<0.001$

$<0.001$

0.10

0.18

0.57

0.85

0.02

0.02

0.10

0.77

0.02

$<0.001$

0.03

0.002

Table 2. 